Louisiana State University

LSU Digital Commons

Faculty Publications

Department of Physics \& Astronomy

3-14-1996

\title{
High-order tunable sum and difference frequency mixing in the xuv region
}

\author{
M. B. Gaarde \\ Lunds Tekniska Högskola \\ P. Antoine \\ Service des Photons Atomes et Molécules
}

A. Persson

Lunds Tekniska Högskola

B. Carré

Service des Photons Atomes et Molécules

A. L'Huillier

Lunds Tekniska Högskola

See next page for additional authors

Follow this and additional works at: https://digitalcommons.Isu.edu/physics_astronomy_pubs

\section{Recommended Citation}

Gaarde, M., Antoine, P., Persson, A., Carré, B., L'Huillier, A., \& Wahlström, C. (1996). High-order tunable sum and difference frequency mixing in the xuv region. Journal of Physics B: Atomic, Molecular and Optical Physics, 29 (5) https://doi.org/10.1088/0953-4075/29/5/006

This Article is brought to you for free and open access by the Department of Physics \& Astronomy at LSU Digital Commons. It has been accepted for inclusion in Faculty Publications by an authorized administrator of LSU Digital Commons. For more information, please contact ir@lsu.edu. 


\section{Authors}

M. B. Gaarde, P. Antoine, A. Persson, B. Carré, A. L'Huillier, and C. G. Wahlström 


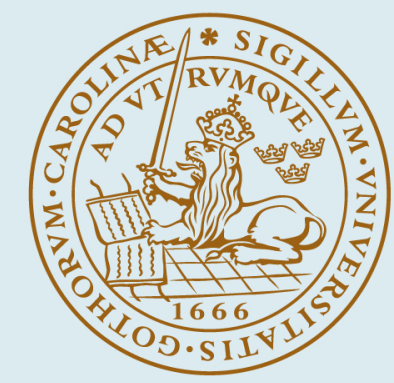

\section{LUND UNIVERSITY}

\section{High-order tunable sum and difference frequency mixing in the xuv region}

Gaarde, Mette; Antoine, P; Persson, Anders; Carre, B; L'Huillier, Anne; Wahlström, ClaesGöran

Published in:

Journal of Physics B: Atomic, Molecular and Optical Physics

DOI:

$10.1088 / 0953-4075 / 29 / 5 / 006$

1996

Link to publication

Citation for published version (APA):

Gaarde, M., Antoine, P., Persson, A., Carre, B., L'Huillier, A., \& Wahlström, C-G. (1996). High-order tunable sum and difference frequency mixing in the xuv region. Journal of Physics B: Atomic, Molecular and Optical Physics, 29(5). https://doi.org/10.1088/0953-4075/29/5/006

Total number of authors:

6

\section{General rights}

Unless other specific re-use rights are stated the following general rights apply:

Copyright and moral rights for the publications made accessible in the public portal are retained by the authors and/or other copyright owners and it is a condition of accessing publications that users recognise and abide by the legal requirements associated with these rights.

- Users may download and print one copy of any publication from the public portal for the purpose of private study or research.

- You may not further distribute the material or use it for any profit-making activity or commercial gain

- You may freely distribute the URL identifying the publication in the public portal

Read more about Creative commons licenses: https://creativecommons.org/licenses/

Take down policy

If you believe that this document breaches copyright please contact us providing details, and we will remove

access to the work immediately and investigate your claim. 
High-order tunable sum and difference frequency mixing in the XUV region

This article has been downloaded from IOPscience. Please scroll down to see the full text article.

1996 J. Phys. B: At. Mol. Opt. Phys. 29 L163

(http://iopscience.iop.org/0953-4075/29/5/006)

View the table of contents for this issue, or go to the journal homepage for more

Download details:

IP Address: 130.235.188.104

The article was downloaded on 08/07/2011 at 07:08

Please note that terms and conditions apply. 


\title{
LETTER TO THE EDITOR
}

\section{High-order tunable sum and difference frequency mixing in the XUV region}

\author{
M B Gaarde $† \S$, P Antoineł, A Persson†, B Carréł, A L’Huillier†‡ and \\ C-G Wahlström $\dagger$ \\ $\dagger$ Division of Atomic Physics, Lund Institute of Technology, PO Box 118, 22100 Lund, Sweden \\ $\ddagger$ Service des Photons, Atomes et Molécules, Centre d’Etudes de Saclay, 91191 Gif sur Yvette, \\ France
}

Received 16 October 1995, in final form 20 November 1995

\begin{abstract}
We present a study of tunable high-order sum and difference frequency mixing in the 7-70 eV range, in xenon, argon and neon. We mix the light from a terawatt, subpicosecond titanium-sapphire laser and that from a tunable optical parametric generator. The radiation generated through mixing processes involves absorption or emission of one or two photons from the weak tunable source. The relative strengths of the sum and difference frequency processes depend strongly on the wavelength range of the generated radiation. The sum frequency processes are stronger at low photon energies in xenon, whereas the difference frequency processes dominate at high photon energies, in neon, being almost comparable to harmonic generation.
\end{abstract}

High-order sum and difference frequency mixing in rare gases has recently attracted much interest, both experimentally (Eichmann et al 1994, 1995, Perry and Crane 1993, Watanabe et al 1994) and theoretically (Eichmann et al 1995, Ivanov et al 1995, Long et al 1995). Most of the experiments have been performed by mixing the light from a high-power short-pulse laser and its second or third harmonic. Depending on the relative intensities and polarizations of the two fields, the odd harmonics can be considerably enhanced (Eichmann et al 1995, Watanabe et al 1994). In the experiments using the second harmonic, even harmonics of the fundamental light are generated with, in some cases, a strength almost comparable to that of the odd-order ones (Perry and Crane 1993). Moreover, the variation of the relative phase of the two fields allows one, in principle, to control harmonic generation in different ways (Ivanov et al 1995, Long et al 1995). Experiments have also been performed by mixing the light from an intense fixed-frequency laser with a tunable light source, leading to tunable radiation in the XUV region. This was first demonstrated by Eichmann and co-workers (1994), using light from a titanium sapphire (Ti:S) laser mixed with tunable radiation from an optical parametric generator (OPG). They generated tunable radiation up to $\sim 31 \mathrm{eV}$, with a tuning range of about $30 \%$ between consecutive harmonics.

There are several advantages of using an OPG for the frequency mixing as compared to using the second or third harmonic. One is, of course, the aspect of tunability, which is of crucial importance for applications. The other is the possibility to identify, and hence to study, sum versus difference frequency generation, since the two processes lead to

$\S$ Also at: Niels Bohr Institute, Oersted Lab., Universitetsparken 5, 2100 Copenhagen, Denmark.

0953-4075/96/050163+06\$19.50 (c) 1996 IOP Publishing Ltd 
different generated wavelengths when the two fields have non-commensurate frequencies. The relative importance of the different frequency conversion mechanisms depends both on the dynamics of an atom in the presence of two fields of different colours, and on phase matching, which makes a clear distinction between sum and difference frequency mixing (Shkolnikov et al 1993, 1995, Bjorklund 1975). On the other hand, since the frequencies of the two fields are non-commensurate, phase control is not possible in this type of experiment.

In this letter, we present a study of high-order sum and difference frequency mixing processes in rare gases, and, in particular, we investigate the difference between the two processes. The experiments are performed by mixing the light from a terawatt, subpicosecond Ti:S laser and that from an OPG pumped by the Ti:S laser. We compare the efficiencies for these mechanisms in different rare gases, i.e. in different conditions of laser intensities and wavelength ranges.

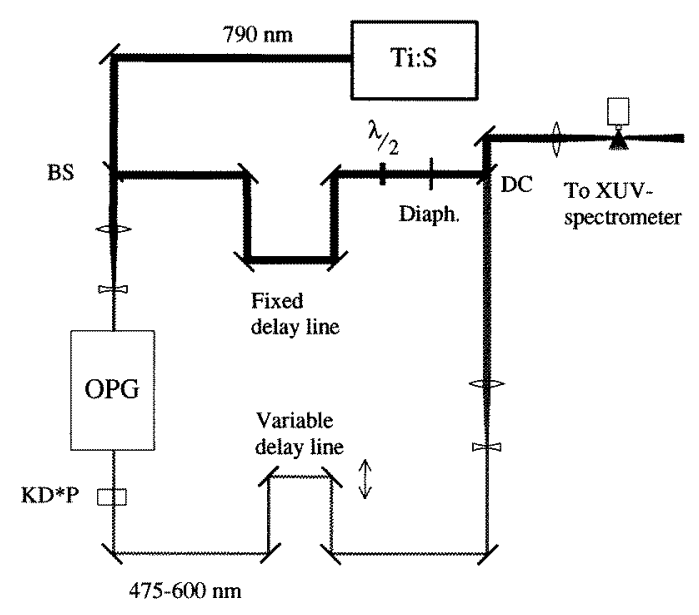

Figure 1. Experimental set-up for sum and difference frequency mixing.

A schematic diagram of the experimental set-up is shown in figure 1. The essence of the set-up is the overlap in space and time of two beams of different colours. The Ti:sapphire laser system, of the DRECAM facility in Saclay, is based on chirped pulse amplification and generates $100 \mathrm{~mJ}, 150 \mathrm{fs}$ pulses at $790 \mathrm{~nm}$ with a $20 \mathrm{~Hz}$ repetition rate. The beam is horizontally polarized and has a diameter of $4 \mathrm{~cm}$. A pulse energy of 60-70 $\mathrm{mJ}$ is used in the present experiment. A beamsplitter, marked as BS in the diagram, directs $25 \%$ of this energy to pump the OPG (Danielius et al 1993), of the Lund high-power laser facility. A telescope just before the OPG reduces the beam diameter to $1 \mathrm{~cm}$ to increase the pump intensity and to match the size of the OPG optics. The non-linear medium in the OPG is a type II BBO ( $\beta$ barium borate) crystal in which radiation from 1150 to $2700 \mathrm{~nm}$ can be produced, by angle tuning, in a three-pass process. Parametric superfluorescence is generated in a first step and is spectrally and spatially narrowed in a second pre-amplification step. The radiation generated is finally amplified in an energy boosting third step. The signal or idler output from the $\mathrm{OPG}$ is mixed with the fundamental radiation in a $\mathrm{KD} * \mathrm{P}$ crystal, and radiation in the 475-600 $\mathrm{nm}$ range can be generated by type II sum-frequency mixing. When the OPG is pumped by $10 \mathrm{~mJ}$ of infrared, the pulse energy of the generated light (green in most of the experiments) is approximately $0.4 \mathrm{~mJ}$. The polarization is vertical.

A variable delay line is installed in the path of the green light to control the temporal overlap necessary for the high-order mixing experiment. A telescope increases the green beam to a diameter of $\sim 5 \mathrm{~cm}$ to decrease the size of the focal spot and hence increase the focused intensity. Moreover, it enables control of the divergence of the green beam, 
which is different from that of the infrared one, and hence improves the spatial overlap in the focus. Well controlled focusing of the green light is difficult due to the limited spatial coherence of the OPG output. Further matching of the green and infrared spot sizes requires that a second telescope reduces the diameter of the infrared beam. This has been used in some of the measurements. The remainder of the pump beam is removed by a dichroic mirror with a $99.9 \%$ reflection at $790 \mathrm{~nm}$, and the infrared signal and idler beams from the OPG are filtered away after a number of reflections of the green light in other dichroic mirrors.

The $75 \%$ of the infrared light not used to pump the OPG is strongly diaphragmed to a diameter of a few $\mathrm{mm}$ and a pulse energy of a few $\mathrm{mJ}$, to maximize the efficiency for harmonic generation and frequency mixing. The polarization is rotated to vertical using a $\lambda / 2$ plate, since parallel polarizations are much more efficient for frequency mixing than perpendicular polarizations (Eichmann et al 1995, Perry and Crane 1993). The two beams, with parallel polarizations, are brought together by a dichroic mirror-indicated as DC in the figure. The beams are focused by the same $f=45 \mathrm{~cm}$ lens into a jet of xenon, argon or neon gas. The intensities in the focus are estimated to be between $10^{14}$ and $10^{15} \mathrm{~W} \mathrm{~cm}^{-2}$ for the infrared and $10^{12}-10^{13} \mathrm{~W} \mathrm{~cm}^{-2}$ for the green light. The radiation generated in the gas jet is analysed with an XUV spectrometer with no entrance slit, utilizing a 700 lines/mm plane grating at grazing incidence and a toroidal mirror. The XUV light is detected with an electron multiplier.

Two spectra obtained in neon are shown in figure 2. The spectra are obtained by stepwise rotation of the grating, and each point is an average of 30 shots. In figure 2(a) the two beams are not overlapped in time-only in space-and hence only odd harmonics of the infrared light are observed with the plateau extending up to approximately $q=37$, and with the highest harmonic $q=45$. The intensity of the green light is not high enough to generate harmonics in this spectral range. The feature between the 35 th and the 37 th harmonic is due to incoherent plasma light. In figure $2(b)$ the two beams are overlapped in time as well, and several additional series of peaks are observed. The peak just to the right

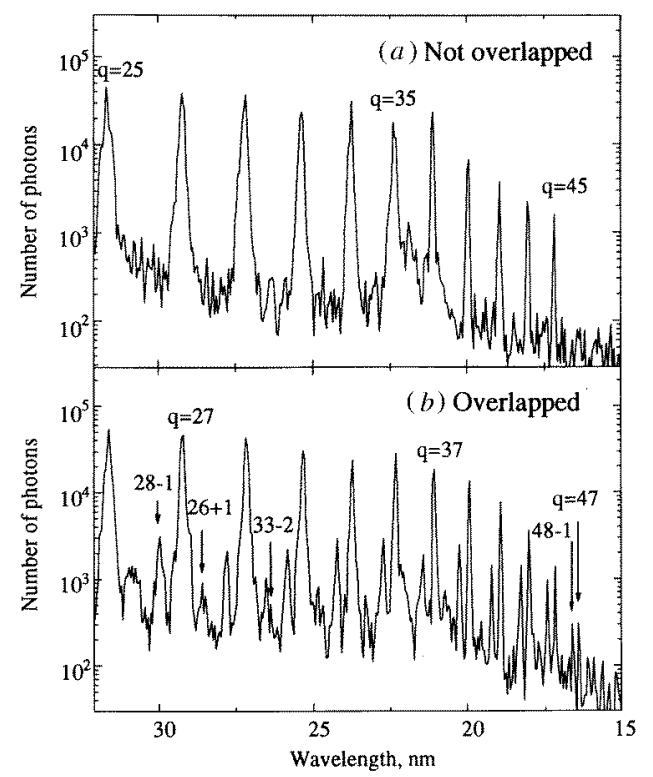

Figure 2. Two spectra obtained in neon. In $(a)$ the two beams are only overlapped in space, whereas in (b) the beams are overlapped in both space and time. The indication $q=27$ refers to the 27 th harmonic of the infrared, and $28-1$ refers to the signal with frequency $28 \omega_{\mathrm{IR}}-\omega_{\mathrm{OPG}}$. 
of the harmonic signal of order $(2 N+1)$ corresponds to a frequency of $2 N \omega_{\mathrm{IR}}+\omega_{\mathrm{OPG}}$ and will be referred to as a $(2 N)+1$. As in harmonic generation, parity conservation requires that the total number of photons absorbed or emitted by the two laser fields is odd. The peak just to the left corresponds to a frequency of $(2 N+2) \omega_{\text {IR }}-\omega_{\text {OPG }}$ and will be referred to as $(2 N+2)-1$. We will, in general, denote sum (respectively difference) frequency processes involving absorption (emission) of one photon from the OPG field by $+1(-1)$. Finally, peaks with frequencies of $(2 N+3) \omega_{\mathrm{IR}}-2 \omega_{\mathrm{OPG}}$ (or, in general, -2$)$ are barely visible between the $(2 N-2)+1$ and the $(2 N+2)-1$ peaks. The mixed signals extend out to $48-1$, which corresponds to a wavelength of $17 \mathrm{~nm}$ or a photon energy of $73 \mathrm{eV}$. The last harmonic is now $q=47$, and when comparing the two spectra, the cut-off seems to be less steep when the green light is present. This is the case in several spectra in neon and argon. Note that the strength of the -1 signals is significantly larger than the +1 signals, and is even approaching that of the harmonic signals at high energies.

In argon, up to the $32-1$ signal has been observed, with a wavelength of $26 \mathrm{~nm}$, or a photon energy of $48 \mathrm{eV}$, and in xenon up to the $20-1$ signal. The efficiency for mixed frequency generation increases from neon to argon, and from argon to xenon, as is the case for harmonic generation. The mixed signals are typically a factor of 10-50 weaker than the corresponding harmonic peaks in the plateau, but are almost comparable in the cut-off region. In xenon, peaks with frequencies $(2 N-1) \omega_{\mathrm{IR}}+2 \omega_{\mathrm{OPG}}$ have been observed, with a strength approximately one order of magnitude lower than the +1 signals.

Tunability is probably the most interesting aspect of frequency mixing for future applications. The present experimental results demonstrate tunability up to $70 \mathrm{eV}$, which extends the previous results of Eichmann et al (1994) quite far into the XUV. With the present tuning range of the $\mathrm{OPG}$, and including \pm 2 processes, i.e. in xenon, $70 \%$ of the spectrum between two subsequent harmonics can be covered, apart from a small region close to the harmonics. At shorter wavelengths, in argon or neon, where 'two-photon' processes are barely detectable, about $40 \%$ of the range can be covered with the \pm 1 signals, leaving an additional gap centred between the two peaks. However, 'two-photon' signals could probably be enhanced in argon and neon as well with a higher OPG energy, which could be obtained by pumping harder. The tuning range could be further extended to cover the entire spectrum between two harmonics by using the frequency-doubled output of the infrared signal from the OPG, instead of the sum-frequency signal generated in the KD*P crystal. An alternative is to change the wavelength of the Ti:S laser to access any desirable tuning range.

Figure 3 presents an investigation of sum versus difference frequency mixing in different wavelength ranges, from xenon with high yields and low photon energies, via argon in the intermediate range, to neon with lower yields and high photon energies. The number of photons produced in each process is plotted as a function of the energy of the emitted radiation. There are several things to be learned from the figure. First, note that the twocolour signals exhibit the same characteristic features as the harmonics, namely a plateau and a cut-off, as also observed in the experiments involving a fundamental and its second or third harmonic (Eichmann et al 1995, Perry and Crane 1993). Since the plateau in neon can extend far beyond what we have observed in this experiment, we believe that the extension of the frequency mixing processes, and thereby the tunability, to the $150 \mathrm{eV}$ range is a question of optimization of the conditions for generation of high-order harmonics. In the quasi-classical two-step interpretation of high-order harmonic generation (Corkum 1993, Schafer et al 1993) an electron is first released by tunnelling ionization, then accelerated by the electric field, before recombining with the nucleus and thereby emitting a harmonic photon. Thus, it is the maximum kinetic energy of the returning electron that determines 

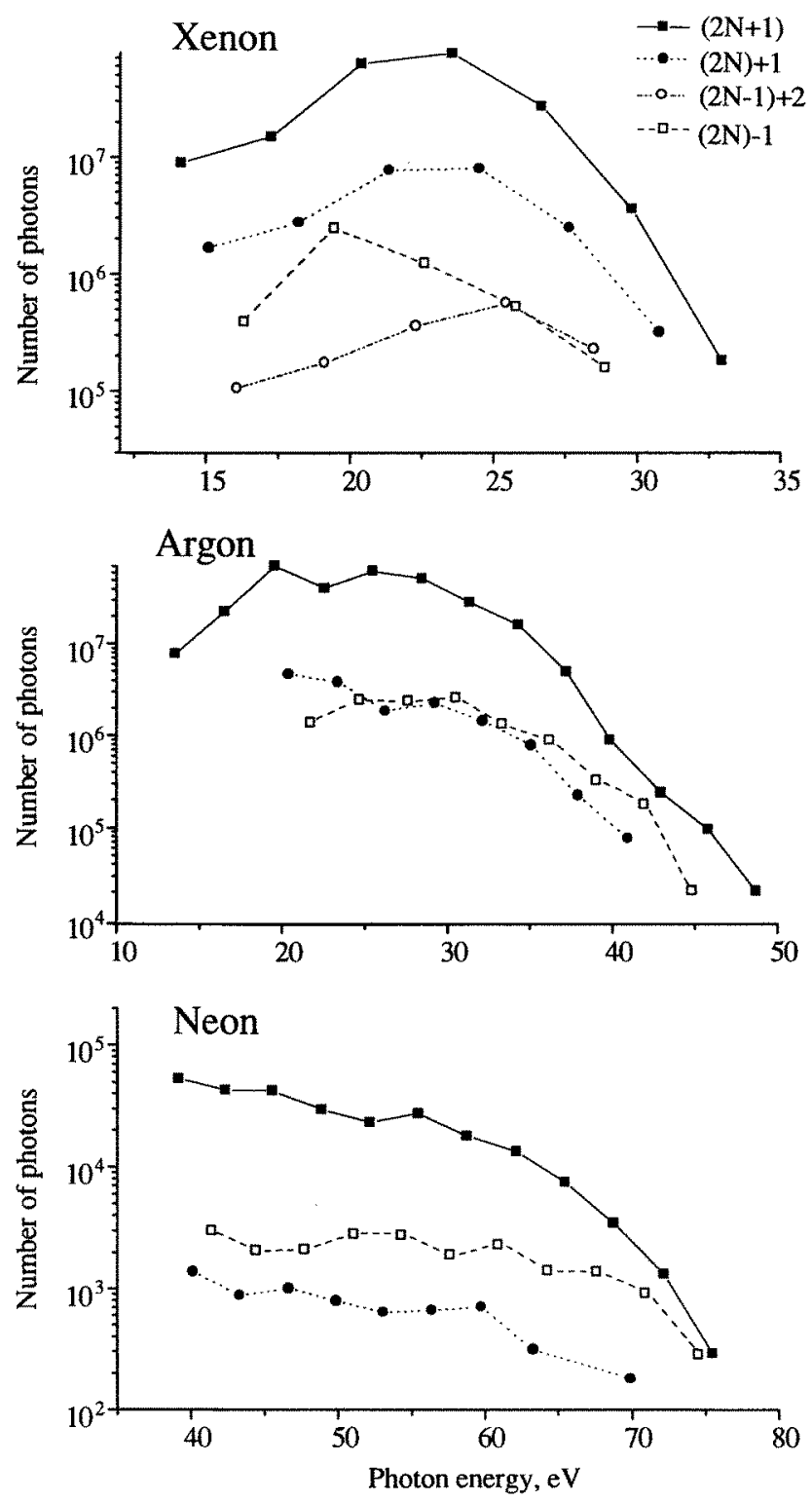

Figure 3. The number of photons as a function of the photon energy, for the different processes in xenon, argon and neon. $(2 N+1)$ refers to the harmonic number $2 N+1$ of the infrared, whereas $(2 N)+1$ refers to the signal with frequency $2 N \omega_{\mathrm{IR}}+\omega_{\mathrm{OPG}}$.

the cut-off. Therefore the two-colour signals are expected to exhibit a similar plateau and cut-off behaviour to one-colour harmonics, as is indeed observed in figure 3. Even the previously noted tendency, that the cut-off is less steep when the green field is present, might be interpreted as an additional acceleration of the electrons by the weak green field.

Second, note the change in the relative strengths of the +1 and -1 processes for increasing photon energies. In xenon, between 15 and $30 \mathrm{eV}$, the +1 signals are clearly dominating, being an order of magnitude stronger than the -1 signals, and the +2 peaks 
are almost as high as the -1 peaks. In argon, between 20 and $40 \mathrm{eV}$, the two processes yield peaks of comparable strengths, the +1 signals being stronger at low energies and the -1 signals taking over at higher energies. Finally, in neon, above $40 \mathrm{eV}$, the -1 signals are dominating the +1 signals by about a factor of 5 and are even approaching the strength of the harmonic signals in the cut-off region. As mentioned previously, -2 peaks are present, barely above the noise level, whereas no +2 peaks can be detected.

At present, we have no explanation for this behaviour. Phase matching is predicted to be more efficient for difference frequency mixing schemes than for sum frequency mixing (Shkolnikov et al 1993, 1995, Bjorklund 1975). Indeed, in difference frequency mixing, the geometrical phase shifts owing to the two colours have opposite signs, and therefore partly compensate, leading to better phase matching. In contrast, in sum frequency processes, the geometrical phase shifts have the same sign and therefore add, leading to poorer phase matching. However, this difference between sum and difference frequency mixing is expected to decrease with the process order (simply, when the number of photons $q$ for the first colour becomes large, $\left.q \phi_{1}+\phi_{2} \sim q \phi_{1} \sim q \phi_{1}-\phi_{2}\right)$. This is at variance with the present experimental observations. The observed behaviour is therefore expected to originate in the single-atom dynamics. A theoretical analysis to interpret the experimental results is in progress.

In conclusion, we have studied sum and difference frequency mixing in the 15-70 $\mathrm{eV}$ range and have observed that the mixed signals behave as harmonic signals, concerning plateau, cut-off energy, and focusing conditions. We observe a marked change in the relative strength of the +1 signals and the -1 signals as the photon energy is increasing. Finally, we believe that the present tunability in the $7-70 \mathrm{eV}$ range can be easily extended to cover the entire range from 7 to $150 \mathrm{eV}$.

We gratefully thank $\mathrm{P}$ D'Oliveira, $\mathrm{P}$ Meynadier and $\mathrm{M}$ Perdrix, of the DRECAM femtosecond laboratory in Saclay, for their helpful support in running the Ti:sapphire laser and setting up the optics for the mixing experiment. The financial support from the Human Capital and Mobility Programme of the European Commission, and the Swedish Natural Science Research Council is acknowledged.

\section{References}

Bjorklund G C 1975 IEEE J. Quantum Electron. QE-11 287

Corkum P B 1993 Phys. Rev. Lett. 711994

Danielius R, Piskarskas A, Persson A and Svanberg S 1993 Lithuanian J. Phys. 33305

Eichmann H, Egbert A, Nolte S, Momma C, Wellegehausen B, Becker W, Long S and McIver J K 1995 Phys. Rev. A 51 R3414

Eichmann H, Meyer S, Riepl K, Momma C and Wellegehausen B 1994 Phys. Rev. A 50 R2834

Ivanov M, Corkum P B, Zuo T and Bandrauk A 1995 Phys. Rev. Lett. 742933

Long S, Becker W and McIver J K 1995 Phys. Rev. A 522262

Perry M D and Crane J K 1993 Phys. Rev. A 48 R4051

Shkolnikov P L, Kaplan A E and Lago A 1993 Opt. Lett. 181700

1995 to be published

Schafer K J, Yang B, DiMauro L F and Kulander K C 1993 Phys. Rev. Lett. 701599

Watanabe S, Kondo K, Nabekawa Y, Sagisaka A and Koboyashi Y 1994 Phys. Rev. Lett. 732692 